\title{
Identification and comprehensive analyses of the CBL and CIPK gene families in wheat (Triticum aestivum L.)
}

Tao Sun ${ }^{\dagger}$, Yan Wang ${ }^{\dagger}$, Meng Wang, Tingting Li, Yi Zhou, Xiatian Wang, Shuya Wei, Guangyuan He* and Guangxiao Yang ${ }^{*}$

\begin{abstract}
Background: Calcineurin B-like (CBL) proteins belong to a unique group of calcium sensors in plant that decode the $\mathrm{Ca}^{2+}$ signature by interacting with CBL-interacting protein kinases (CIPKs). Although CBL-CIPK complexes have been shown to play important roles in the responses to various stresses in plants, little is known about their functions in wheat.

Results: A total of seven TaCBL and 20 TaCIPK genes were amplified from bread wheat, Triticum aestivum cv. Chinese Spring. Reverse-transcriptase-polymerase chain reaction (RT-PCR) and in silico expression analyses showed that TaCBL and TaCIPK genes were expressed at different levels in different tissues, or maintained at nearly constant expression levels during the whole life cycle of the wheat plant. Some TaCBL and TaCIPK genes showed up- or down-regulated expressions during seed germination. Preferential interactions between TaCBLs and TaCIPKs were observed in yeast two-hybrid and bimolecular fluorescence complementation experiments. Analyses of a deletion series of TaCIPK proteins with amino acid variations at the C-terminus provided new insights into the specificity of the interactions between TaCIPKs and TaCBLs, and indicated that the TaCBL-TaCIPK signaling pathway is very complex in wheat because of its hexaploid genome. The expressions of many TaCBLS and TaCIPKs were responsive to abiotic stresses (salt, cold, and simulated drought) and abscisic acid treatment. Transgenic Arabidopsis plants overexpressing TaCIPK24 exhibited improved salt tolerance through increased $\mathrm{Na}^{+}$efflux and an enhanced reactive oxygen species scavenging capacity.
\end{abstract}

Conclusions: These results contribute to our understanding of the functions of CBL-CIPK complexes and provide the basis for selecting appropriate genes for in-depth functional studies of CBL-CIPK in wheat.

Keywords: TaCIPK24, CBL-CIPK, Expression profiles, Stress response, Preferential interactions, Wheat

\section{Background}

The calcium ion $\left(\mathrm{Ca}^{2+}\right)$ functions as a ubiquitous second messenger in plants, and participates in signal transduction in response to various environmental and developmental stimuli. The transient $\mathrm{Ca}^{2+}$ signal in the cytoplasm of plant cells is captured by a variety of $\mathrm{Ca}^{2+}$ sensors including calmodulins, calmodulin-like proteins (CML), calciumdependent protein kinases (CDPKs), and calcineurin B-like

\footnotetext{
*Correspondence: hegy@hust.edu.cn; ygx@hust.edu.cn

'Equal contributors

The Genetic Engineering International Cooperation Base of Chinese Ministry of Science and Technology, The Key Laboratory of Molecular Biophysics of Chinese Ministry of Education, College of Life Science and Technology, Huazhong University of Science \& Technology, Wuhan 430074, China
}

(CBL) proteins [1-3]. These $\mathrm{Ca}^{2+}$ sensors transfer the environmental and developmental signals to downstream components, causing a series of physiological and biochemical changes.

CBLs, which harbor a core region consisting of four conserved $\mathrm{EF}$ hands, capture the $\mathrm{Ca}^{2+}$ signal, and interact with CBL-interacting protein kinases (CIPKs) to phosphorylate their target proteins [4]. The CIPKs with the NAF motif for interaction with CBL belong to the SnRK3 protein family, whose members have a specific Ser/Thr protein kinase domain [5]. Recently, CIPK proteins were shown to phosphorylate their interacting CBL proteins at N-terminal conservative Ser residue (AtCBL1-Ser ${ }^{201}$, AtCBL2-Ser ${ }^{216}$, 
AtCBL4-Ser ${ }^{205}$ and AtCBL10-Ser ${ }^{237}$ ) [6-8] and this proper phosphorylation of CBL was proved to be absolutely required for the in vivo activation of the AKT1 $\mathrm{K}^{+}$channel by CBL1-CIPK23 and CBL9-CIPK23 complexes in oocytes [6]. The $C B L$ and $C I P K$ gene families have been described in Arabidopsis thaliana, Oryza sativa, Zea mays, and Brassica napus [9-12].

In previous studies, CBL-CIPK complexes were found to play very important roles in responses to external stimuli. The salt overly sensitive (SOS) pathway was the first CBL-CIPK network to be identified, and this CBLCIPK complex comprised CBL4 (SOS3) and CIPK24 (SOS2). The CBL4 protein was shown to interact with CIPK24 and recruit it to the cytoplasmic membrane, where it activated the $\mathrm{H}^{+} / \mathrm{Na}^{+}$(SOS1) antiporter to enhance salt tolerance [13]. This result indicated that the CBL-CIPK complex functions in regulating sodium ion homeostasis. Meanwhile, V-ATPase proteins were shown to directly interact with SOS2 to rescue the salt-defective phenotype of $\operatorname{sos} 2$ mutant plants [14]. In recent years, many CBLs and CIPKs have been shown to promote stress tolerance by balancing the intracellular ion concentration in plants. The sodium concentration was markedly lower in the Arabidopsis cbl10 mutant than in control plants, in both normal and salt-stress conditions [15]. The CBL10-overexpressing lines exhibited a $\mathrm{K}^{+}$-sensitive phenotype, like that of the akt1 mutant under low $\mathrm{K}^{+}$conditions. The interaction between CBL10 and AKT1 was verified in bimolecular fluorescence complementation (BiFC), yeast two-hybrid $(\mathrm{Y} 2 \mathrm{H})$, and co-immunoprecipitation experiments, and was shown to impair AKT1-mediated inward potassium $\left(\mathrm{K}^{+}\right)$ currents [16]. AtCIPK23 could directly interact with CBL1 to promote $\mathrm{K}^{+}$uptake under low $\mathrm{K}^{+}$conditions by phosphorylating and activating the $\mathrm{K}^{+}$channel (AKT1) in Arabidopsis and rice [17, 18]. However, a recent study suggested that CBL1/CBL9 might interact with AKT1 independently of CIPK23 [19]. Moreover, in response to low- $\mathrm{K}^{+}$conditions, CIPK9 was recruited to the tonoplast by CBL3 to regulate $\mathrm{K}^{+}$homeostasis in Arabidopsis [20].

Over-expression and mutant analyses of CBLs and CIPKs have greatly enriched our understanding of their functions. The $c b l 2 / c b l 3$ double mutant showed defects in growth and ion homeostasis, possibly as a result of disrupted vacuolar $\mathrm{H}^{+}$-ATPase activity [21]. In another study, CBL2 and CBL3 were shown to affect seed development and morphology [22]. The ectopic overexpression of ZmCIPK16 in the Arabidopsi sos 2 mutant enhanced the expression of SOS1 and improved salt tolerance under saline conditions [12]. AtCIPK3 was induced by abscisic acid (ABA) and stress conditions and established a molecular connection between stress- and ABA-induced $\mathrm{Ca}^{2+}$ signals [23]. CBL10 and CIPK6 were shown to positively regulate both immunity- and disease-associated programmed cell death in tomato and tobacco [24]. The oscipk31 insertion mutant was hypersensitive to $\mathrm{ABA}$ and stresses (salt, mannitol, and glucose), and showed altered expression levels of several stress-responsive genes when it was exposed to these abiotic stresses during the seed germination and seedling stages [25]. In a recent study, a few AtCBL members were also found to target to another group of enzymes 5'-methylthioadenosine nucleosidases by yeast two-hybrid system, indicting an additional level of complexity on the CBL-mediated signaling networks [26]. However, with respect to the larger families of CBLs and CIPKs, few CBL-CIPK complexes have been functionally characterized, especially in bread wheat (Triticum aestivum L.), which is one of the most important food crops. Bread wheat has an allohexaploid genome with a size of approximately $17 \mathrm{~GB}$, making it one of the largest and most complex plant genomes. In recent years, the genome sequencing of T. aestivum, Triticum urartu, and Aegilops tauschii has promoted research on bread wheat, especially studies on agronomically important gene families related to disease resistance, abiotic stress endurance, and grain quality [27-29].

In our previous work, we demonstrated that transgenic tobacco overexpressing wheat TaCIPK14 and TaCIPK29 showed increased tolerance to salinity and drought stress, respectively $[30,31]$. In this study, seven $T a C B L$ and 20 TaCIPK genes in wheat were identified and cloned by a genome-wide analysis combined with expressed sequence tag (EST) assembly. Here, we present the results of analyses of expression profiles, the specific interactions between TaCBLs and TaCIPKs, and the stress tolerance of transgenic Arabidopsis plants expressing TaCIPK24.

\section{Results and discussion}

Identification of TaCBL and TaCIPK gene families in wheat The bread wheat genome is thought to have formed via fusion of three ancestral genomes, which are believed to be related to T. urartu, Aegilops speltoides, and A. tauschii. These fusion events are thought to have occurred several hundred thousand years ago [32]. We searched the wheat genome and found 24 CBL (7, 9, and 8 loci in sub-genomes $\mathrm{A}, \mathrm{B}$, and D, respectively) and 79 CIPK $(21,34$, and 24 loci in sub-genomes $\mathrm{A}, \mathrm{B}$, and $\mathrm{D}$, respectively) loci on wheat chromosomes (Table 1). The relative positions of these loci on chromosomes were identified on genetic maps (Additional file 1). The protein and DNA sequences of T. urartu and A. tauschii (the B sub-genome was not analyzed in this work) were retrieved from NCBI and all the $C B L$ and $C I P K$ nucleotide sequences from these three species (T. aestivum, T. urartu, and A. tauschii) were clustered against each other (Fig. 1). The closest matching genes were regarded as equivalent partners, 
Table 1 Identification of TaCBL and TaCIPK gene families in wheat genome

\begin{tabular}{|c|c|c|c|c|c|c|c|c|c|c|}
\hline \multirow{2}{*}{$\begin{array}{l}\text { Gene }^{a} \\
\text { TaCBL1 }\end{array}$} & \multirow{2}{*}{$\begin{array}{l}\text { Gene names related to } \mathrm{Chr} \text {. } \\
\text { TaCBL1-A1 }\end{array}$} & \multirow{2}{*}{$\begin{array}{l}\text { Amino Acid } \\
215\end{array}$} & \multirow{2}{*}{$\begin{array}{l}\text { Exon } \\
8\end{array}$} & \multirow{2}{*}{$\begin{array}{l}\text { Chr. } \\
1 \mathrm{AL}\end{array}$} & \multirow{2}{*}{$\begin{array}{l}\text { Contigs } \\
3974931\end{array}$} & \multicolumn{2}{|c|}{$\begin{array}{l}\text { Gene Position } \\
\text { Start end }\end{array}$} & \multirow{2}{*}{$\begin{array}{l}\text { Triticum urartu } \\
\text { KD278069 }\end{array}$} & \multirow{2}{*}{$\begin{array}{l}\text { Aegilops tauschii } \\
-\end{array}$} & \multirow{2}{*}{$\begin{array}{l}\text { GenBank Acc No. } \\
\text { JX2443002 }\end{array}$} \\
\hline & & & & & & 2941 & 6756 & & & \\
\hline & $\operatorname{TaCBL1} 1-A 2^{p}$ & - & - & & - & - & - & KD162834 & & \\
\hline & $T a C B L 1-B$ & - & - & $1 \mathrm{BL}$ & 3900149 & 166 & 3357 & - & - & \\
\hline & TaCBL1-D1 & - & - & $1 \mathrm{DL}$ & 1737972 & 560 & 1484 & - & KD512543 & \\
\hline & TaCBL1-D2 & - & - & $1 \mathrm{DL}$ & 464815 & 15 & & - & KD578957 & \\
\hline \multirow[t]{3}{*}{$T a C B L 2^{a}$} & $T a C B L 2-A$ & 215 & 8 & $5 A S$ & 1464783 & 5151 & 93449 & KD141203 & - & $J \times 243003$ \\
\hline & $T a C B L 2-B^{*}$ & 245 & 7 & $5 \mathrm{BS}$ & 2281375 & 80 & 4667 & - & - & \\
\hline & $T a C B L 2-D$ & 225 & - & $5 D S$ & 2741958 & 5680 & 9158 & - & KD544824 & \\
\hline \multirow[t]{3}{*}{$\operatorname{TaCBL} 3^{\text {a }}$} & TaCIPK3-A & - & - & - & - & - & - & KD241885 & - & JX243004 \\
\hline & $T a C B L 3-B^{*}$ & - & 8 & $4 \mathrm{BS}$ & 4897686 & 4127 & 8100 & - & - & \\
\hline & TaCBL3-D & 226 & - & $4 \mathrm{DS}$ & 2274776 & 2834 & 7044 & - & KD515143 & \\
\hline \multirow[t]{4}{*}{$\operatorname{TaCBL} 4^{\mathrm{a}}$} & $T a C B L 4-A$ & - & - & $1 \mathrm{AL}$ & 3951410 & 100 & 1380 & KD20084 & - & JX243005 \\
\hline & $T a C B L 4-B$ & - & 7 & $1 \mathrm{BL}$ & 3917583 & 1507 & 2702 & - & - & \\
\hline & $\operatorname{TaCBL4-D1^{*}}$ & 218 & - & $1 \mathrm{DL}$ & 2274485 & 1479 & 2868 & - & KD597121 & \\
\hline & $T a C B L 4-D 2$ & - & 7 & $1 \mathrm{DL}$ & 2273437 & 4080 & 5129 & - & KD604528 & \\
\hline \multirow[t]{3}{*}{ TaCBL6 ${ }^{a}$} & TaCBL6-A & 218 & 5 & $5 \mathrm{AL}$ & 2189286 & 246 & 2332 & KD045461 & - & JX243006 \\
\hline & TaCBL6-B & 226 & 8 & $5 B \mathrm{~B}$ & 10906286 & 5260 & 8935 & - & - & \\
\hline & TaCBL6-D & - & - & $5 \mathrm{DL}$ & 4595540 & 1995 & 5159 & - & KD546381 & \\
\hline \multirow[t]{3}{*}{$T a C B L 7^{a}$} & TaCBL7-A & - & - & $1 \mathrm{AL}$ & 3887581 & 5948 & 7521 & KD220101 & - & JX243001 \\
\hline & $T a C B L 7-B^{*}$ & - & 8 & $1 \mathrm{BL}$ & 3897439 & 22549 & 24230 & - & - & \\
\hline & TaCBL7-D & 213 & 8 & $1 \mathrm{DL}$ & 2195979 & 3589 & 5243 & - & KD592851 & \\
\hline \multirow[t]{7}{*}{ TaCBL9 a } & TaCBL9-A1 & 296 & 8 & $3 \mathrm{AL}$ & 4339532 & 1 & 784 & KD258230 & - & JX243010 \\
\hline & $T a C B L 9-D 1^{P}$ & - & - & $3 D$ & - & - & - & - & KD508651 & \\
\hline & TaCBL9-A2 & - & 5 & $3 A S$ & 3303239 & 2125 & 4252 & KD217616 & - & \\
\hline & $T a C B L 9-D 2^{P}$ & 296 & - & - & - & - & - & - & KD546760 & \\
\hline & $T a C B L 9-B 1^{*}$ & 296 & 9 & $3 B$ & 10535092 & 2016 & 4459 & - & - & \\
\hline & $T a C B L 9-B 2$ & - & - & $3 B$ & 10578811 & 5421 & 7996 & - & - & \\
\hline & TaCBL9-B3 & - & - & $3 B$ & 10423444 & 3743 & 5534 & - & - & \\
\hline \multirow[t]{3}{*}{ TaCIPK2 ${ }^{a}$} & TaCIPK2-A & - & 1 & $2 A S$ & 1136699 & 1058 & 2612 & KD178374 & - & KJ561791 \\
\hline & TaCIPK2-B & 452 & 1 & $2 \mathrm{BS}$ & 5209173 & 4420 & 6031 & - & - & \\
\hline & TaCIPK2-D* & 452 & 1 & 2DS & 5352995 & 3439 & 6170 & - & KD537702 & \\
\hline \multirow[t]{3}{*}{ TaCIPK3 ${ }^{a}$} & TaCIPK3-A & 456 & 14 & $2 \mathrm{AS}$ & 5278308 & 778 & 4232 & KD224079 & - & KJ561800 \\
\hline & TaCIPK3-B & 447 & 14 & $2 \mathrm{BS}$ & 5215259 & 1889 & 4792 & - & - & \\
\hline & TaCIPK3-D* & 447 & 14 & 2DS & 5388639 & 6194 & 9544 & - & KD551511 & \\
\hline \multirow[t]{2}{*}{ TaCIPK4 } & TaCIPK4-B & 448 & 22 & $5 \mathrm{BS}$ & 2276618 & 1805 & 3100 & - & - & - \\
\hline & TaCIPK4-D & 432 & & 5DS & 2735985 & 15903 & 17161 & - & KD505215 & \\
\hline \multirow[t]{3}{*}{ TaCIPK5 a } & TaCIPK5-A & 433 & 1 & $3 A S$ & 3302816 & 1652 & 3448 & KD141920 & - & KJ561802 \\
\hline & TaCIPK5-B & 466 & 1 & $3 B$ & 10642252 & 4923 & 6712 & - & - & \\
\hline & TaCIPK5-D* & 464 & - & 3DS & 1035659 & 1 & 1176 & - & KD508285 & \\
\hline \multirow[t]{2}{*}{ TaCIPK6 } & TaCIPK6- $A^{P}$ & - & - & - & - & & & KD207883 & - & - \\
\hline & TaCIPK6-D $D^{P}$ & - & - & - & - & & & - & KD557345 & \\
\hline \multirow[t]{2}{*}{$\operatorname{TaCIPK} 7^{\mathrm{a}}$} & TaCIPKT-A1 ${ }^{P}$ & - & - & - & - & & & KD036546 & & KJ561803 \\
\hline & $\mathrm{TaCIPK} 7-A 2^{P}$ & - & - & - & - & & & KD051070 & & \\
\hline
\end{tabular}


Table 1 Identification of TaCBL and TaCIPK gene families in wheat genome (Continued)

\begin{tabular}{|c|c|c|c|c|c|c|c|c|c|c|}
\hline & $T a C I P K 7-A 3^{P}$ & - & - & - & - & & & KD103566 & & \\
\hline & TaCIPK7-B & - & 1 & $5 B L$ & 10732661 & 4349 & 5764 & - & - & \\
\hline & TaCIPK7-D $D^{P}$ & 431 & - & - & - & & & - & KD522041.1 & \\
\hline \multirow[t]{2}{*}{ TaCIPK8 ${ }^{a}$} & TaCIPK $8-B$ & - & 3 & $3 B$ & 10524427 & 1670 & 3978 & - & - & KJ561804 \\
\hline & TaCIPK8-D & 464 & - & $3 \mathrm{DL}$ & 6956205 & 367 & 2132 & - & - & \\
\hline \multirow[t]{3}{*}{ TaCIPK9 } & TaCIPK9-A & - & 14 & $5 \mathrm{AL}$ & 1668033 & 412 & 3585 & KD006963 & - & AK332473 \\
\hline & TaCIPK9-B & 446 & - & $4 \mathrm{BL}$ & 6995603 & 4780 & 8912 & - & - & \\
\hline & TaCIPK9-D & - & 15 & $4 \mathrm{DL}$ & 14472121 & & & - & KD554016 & \\
\hline \multirow[t]{3}{*}{ TaCIPK10 a } & TaCIPK10-A & 445 & 1 & $4 \mathrm{AS}$ & 5974107 & 2247 & 3728 & KD248893 & - & KJ561787 \\
\hline & TaCIPK10-B & 384 & 1 & $4 \mathrm{BL}$ & 7036526 & 5067 & 6516 & - & - & \\
\hline & TaCIPK10-D* & 439 & 1 & $4 \mathrm{DL}$ & 14429928 & & & - & KD540780 & \\
\hline \multirow[t]{5}{*}{ TaCIPK $11^{\mathrm{a}}$} & TaCIPK11-A & 438 & 1 & $3 \mathrm{AL}$ & 2838468 & 1233 & 3144 & KD051997 & - & KJ561788 \\
\hline & TaCIPK11-B1 & 507 & 1 & $3 B$ & 10507835 & 1 & 1663 & - & - & \\
\hline & TaCIPK11-B2 & 507 & - & $3 B$ & 10507836 & 1 & 1544 & - & - & \\
\hline & TaCIPK11-D & - & 1 & $3 \mathrm{DL}$ & 6897880 & 8953 & 10856 & - & KD509836 & \\
\hline & TaCIPK11-D2 & 507 & - & & & & & & KD556601 & \\
\hline \multirow[t]{3}{*}{ TaCIPK12 } & TaCIPK12-A & - & 2 & $1 \mathrm{AL}$ & 3875215 & 1 & 875 & KD167973 & - & - \\
\hline & TaCIPK12-B & - & 1 & $1 \mathrm{BL}$ & 3828145 & 1 & 326 & - & - & \\
\hline & TaCIPK12-D & - & 1 & $1 \mathrm{DL}$ & 2112699 & 1 & 418 & - & KD507283 & \\
\hline \multirow[t]{2}{*}{ TaCIPK13 } & TaCIPK13-B & - & 1 & $3 B$ & 10753103 & & & - & - & - \\
\hline & TaCIPK13-D $D^{P}$ & - & - & - & - & - & - & - & KD502264 & \\
\hline \multirow[t]{3}{*}{ TaCIPK 14} & TaCIPK14-A & - & 1 & $4 \mathrm{AL}$ & 7147326 & & & KD239100 & - & JX879754 \\
\hline & TaCIPK 14-B & 449 & 1 & $4 \mathrm{BS}$ & 4951408 & 11612 & 13239 & - & - & \\
\hline & TaCIPK14-D & 444 & 1 & $4 \mathrm{DS}$ & 2283949 & 3085 & 4712 & - & KD546797 & \\
\hline \multirow[t]{3}{*}{ TalPK15 a } & TalPK15-A & 449 & 1 & $5 \mathrm{AL}$ & 1668033 & 1866 & 3335 & - & - & KJ561789 \\
\hline & TaCIPK15-B & - & $1-$ & $5 B \mathrm{BL}$ & 10796477 & 2838 & 4331 & - & - & \\
\hline & TaCIPK15-D* & 438 & & $5 \mathrm{DL}$ & 4490016 & 1021 & 2355 & - & KD560960 & \\
\hline \multirow[t]{3}{*}{ TaCIPK16 } & TaCIPK16- $A^{P}$ & - & - & - & - & - & - & KD143696 & - & AK331419 \\
\hline & TaCIPK16-B & - & 2 & $5 B \mathrm{~B}$ & 10860020 & 4595 & 6233 & - & - & \\
\hline & TaCIPK16-D $D^{P}$ & 447 & - & - & - & - & - & - & KD721304 & \\
\hline \multirow[t]{7}{*}{ TaCIPK $17^{\mathrm{a}}$} & TaCIPK17-A1 & - & 6 & 1AS & 3313233 & 19 & 2337 & KD250230 & - & KJ561790 \\
\hline & TaCIPK17-A2 & - & 11 & $1 \mathrm{AS}$ & 3259469 & 1075 & 3521 & KD132920 & - & \\
\hline & TaCIPK17-B1 & - & 12 & $1 \mathrm{BS}$ & 1265003 & 1916 & 5673 & - & - & \\
\hline & TaCIPK17-B2 & 466 & 12 & $1 \mathrm{BS}$ & 3424219 & 1678 & 5957 & - & - & \\
\hline & TaCIPK17-D1 & 466 & - & $1 \mathrm{DS}$ & 1916039 & 456 & 3542 & - & KD530641 & \\
\hline & TaCIPK17-D2 & - & - & $1 \mathrm{DS}$ & 1890755 & 1 & 3079 & - & KD563756 & \\
\hline & TaCIPK17-D3 & - & 11 & 1DS & 271737 & 999 & 3311 & - & KD589116 & \\
\hline \multirow[t]{3}{*}{ TaCIPK $19^{a}$} & TaCIPK19-A $A^{P}$ & - & - & - & - & - & - & KD117636 & - & JX234011 \\
\hline & TaCIPK19-B1 & - & 2 & $3 B$ & 10573976 & 10157 & 13650 & - & - & \\
\hline & TaCIPK19-B2 & 483 & 1 & $3 B$ & 10753103 & 13446 & 14093 & - & - & \\
\hline \multirow[t]{3}{*}{ TaCIPK20 } & TaCIPK20-A & 525 & 4 & $6 \mathrm{AS}$ & 4406549 & & & KD224235 & - & - \\
\hline & TaCIPK20-B & - & 12 & $6 \mathrm{BS}$ & 2936057 & & & - & - & \\
\hline & TaCIPK20-D & - & 11 & $6 \mathrm{DS}$ & 2096196 & & & - & KD544165 & \\
\hline \multirow[t]{2}{*}{ TaCIPK $21^{\text {a }}$} & TaCIPK21-A & - & 12 & $2 A S$ & 5304926 & 1845 & 6080 & KD277172 & - & KJ561792 \\
\hline & TaCIPK21-B & 451 & - & $2 \mathrm{BS}$ & 5197551 & 4370 & 8637 & - & - & \\
\hline
\end{tabular}


Table 1 Identification of TaCBL and TaCIPK gene families in wheat genome (Continued)

\begin{tabular}{|c|c|c|c|c|c|c|c|c|c|c|}
\hline & TaCIPK21-D & - & - & $2 \mathrm{DS}$ & 5319123 & 2007 & 4463 & - & KD511088 & \\
\hline \multirow[t]{6}{*}{ TaCIPK22 ${ }^{a}$} & TaCIPK22-A & - & - & $2 \mathrm{AL}$ & 6383902 & 1 & 934 & KD203757 & - & KJ561793 \\
\hline & TaCIPK22-B1 & - & - & $2 \mathrm{BL}$ & 7988831 & 2848 & 4399 & - & - & \\
\hline & TaCIPK22-B2 & - & - & $2 \mathrm{BL}$ & 8033849 & 3684 & 4533 & - & - & \\
\hline & TaCIPK22-B3 & - & - & $2 \mathrm{BL}$ & 7949729 & 3040 & 4484 & - & - & \\
\hline & TaCIPK22-D1 & - & 1 & $2 \mathrm{DL}$ & 9860188 & 4959 & 6607 & - & KD500669 & \\
\hline & TaCIPK22-D2 & 433 & - & $2 \mathrm{DL}$ & 9908802 & 3988 & 4888 & - & - & \\
\hline \multirow[t]{3}{*}{ TaCIPK23 } & TaCIPK23-A & - & 14 & $2 \mathrm{AL}$ & 6369709 & 442 & 4149 & KD007171 & - & $J \times 243012$ \\
\hline & TaCIPK23-B & 452 & 14 & $2 \mathrm{BL}$ & 7939869 & 5925 & 8812 & - & - & \\
\hline & TaCIPK23-D & 462 & 14 & $2 \mathrm{DL}$ & 9894674 & 10517 & 14199 & - & KD502068 & \\
\hline \multirow[t]{4}{*}{ TaCIPK24 ${ }^{a}$} & TaCIPK24-A & 462 & - & $7 A L$ & 4552082 & 7689 & 11571 & KD185370 & - & KJ561794 \\
\hline & TaCIPK24-B1 & - & - & $7 \mathrm{BL}$ & 6743884 & 3133 & 8220 & - & - & \\
\hline & TaCIPK24-B2 & - & - & $7 B L$ & 6725100 & 299 & 1430 & - & - & \\
\hline & TaCIPK24-D & - & 14 & $7 D L$ & 3369425 & 6709 & 12113 & - & KD554191 & \\
\hline \multirow[t]{2}{*}{ TaCIPK25 a } & TaCIPK25-A & 446 & - & $7 \mathrm{AL}$ & 4557464 & 1 & 906 & KD203605 & - & KJ561795 \\
\hline & TaCIPK25-D* & - & - & $7 D L$ & 3315123 & 3089 & 4703 & - & KD578984 & \\
\hline \multirow[t]{3}{*}{ TaCIPK26 ${ }^{\mathrm{a}}$} & TaCIPK26-A & - & 1 & $6 \mathrm{AS}$ & 4399246 & 659 & 2242 & - & - & KJ561796 \\
\hline & TaCIPK26-B & 481 & - & $6 \mathrm{BS}$ & 1542240 & 652 & 2262 & - & - & \\
\hline & TaCIPK26-D & - & 1 & $6 \mathrm{DS}$ & 32939 & 832 & 2285 & - & KD556601 & \\
\hline \multirow[t]{2}{*}{ TaCIPK27 ${ }^{\mathrm{a}}$} & TaCIPK27-B1 & 486 & - & $5 B \mathrm{BL}$ & 10894042 & 2308 & 3872 & - & - & KJ561797 \\
\hline & TaCIPK27-B2 & - & 1 & $5 B \mathrm{~B}$ & 10894043 & 2308 & 3872 & - & - & \\
\hline \multirow[t]{3}{*}{ TaCIPK28 ${ }^{a}$} & $\operatorname{TaCIPK} 28-A^{*}$ & 447 & 1 & $1 \mathrm{AL}$ & 2752572 & 4061 & 5848 & KD259754 & - & KJ561798 \\
\hline & TaCIPK28-B & 472 & 1 & $1 \mathrm{BL}$ & 3799843 & 6758 & 8542 & - & - & \\
\hline & TaCIPK28-D & 472 & 1 & $1 \mathrm{DL}$ & 2281163 & 487 & 2269 & - & KD506845 & \\
\hline \multirow[t]{3}{*}{ TaCIPK29 a } & TaCIPK29-A $A^{P}$ & 472 & - & & - & - & - & KD176996 & - & JX243013 \\
\hline & TaCIPK29-B* & - & 1 & $2 \mathrm{BS}$ & 5246729 & 19006 & 20606 & - & - & \\
\hline & TaCIPK29-D $D^{P}$ & 436 & - & - & - & - & - & - & KD506473 & \\
\hline TaCIPK30 & TaCIPK30-A & - & 1 & $3 \mathrm{AL}$ & 4303945 & 2592 & 3265 & KD117636 & - & AK330597 \\
\hline \multirow[t]{3}{*}{ TaCIPK31 a } & TaCIPK31-A & 478 & 14 & $4 \mathrm{AS}$ & 5932853 & 800 & 4412 & KD170661 & - & KJ561799 \\
\hline & TaCIPK31-B & 449 & 15 & $4 \mathrm{BL}$ & 7038614 & 5874 & 10593 & - & - & \\
\hline & TaCIPK31-D $D^{P}$ & 449 & - & - & - & - & - & - & KD566562 & \\
\hline \multirow[t]{3}{*}{ TaCIPK32 a } & TaCIPK32-A & - & - & - & - & - & - & KD060684 & - & KJ561801 \\
\hline & TaCIPK32-B & - & 14 & $4 \mathrm{BS}$ & 4875486 & 20714 & 24433 & - & - & \\
\hline & TaCIPK32-D & 439 & 14 & 4DS & 2325367 & 3990 & 77792 & - & KD929083 & \\
\hline
\end{tabular}

"-" represents no data available. " $\mathrm{P}$ " represents the potential genes were not identified in this work. "a" represents those genes that were amplified in this study. "*" represents those amplified genes that were identified as transcripts from indicated chromosomes

as shown in Table 1. Ten and nine genes of T. urartu and A. tauschii, respectively, had no equivalents in T. aestivum, implying the existence of potential un-identified $T a C B L /$ TaCIPK genes. Additionally, TaCIPK15-A, TaCIPK26- $A$, TaCIPK8-D, and TaCIPK22-D2 (wheat specific genes) had no equivalents in $T$. urartu and $A$. tauschii (Table 1 ). Considering that the physical maps of current wheat genome do not cover all the chromosome arms, it is difficult to know whether these potential un-identified $\mathrm{TaCBL} / \mathrm{TaCIPK}$ genes are resulted from gene retention, duplication and whether these wheat specific genes are the results of the loss of $C B L$ and $C I P K$ genes after polyploidization. In the future, a more detailed wheat physical map may resolve this question.

Among the identified TaCBL/TaCIPK genes, the cDNAs of seven TaCBLs and 20 TaCIPKs were successfully PCR amplified from hexaploid bread wheat (T. aestivum) using gene-specific primers (Additional file 2) from a cDNA mixture prepared from wheat cv. Chinese Spring as the template. The genomic DNA and cDNA of all identified 


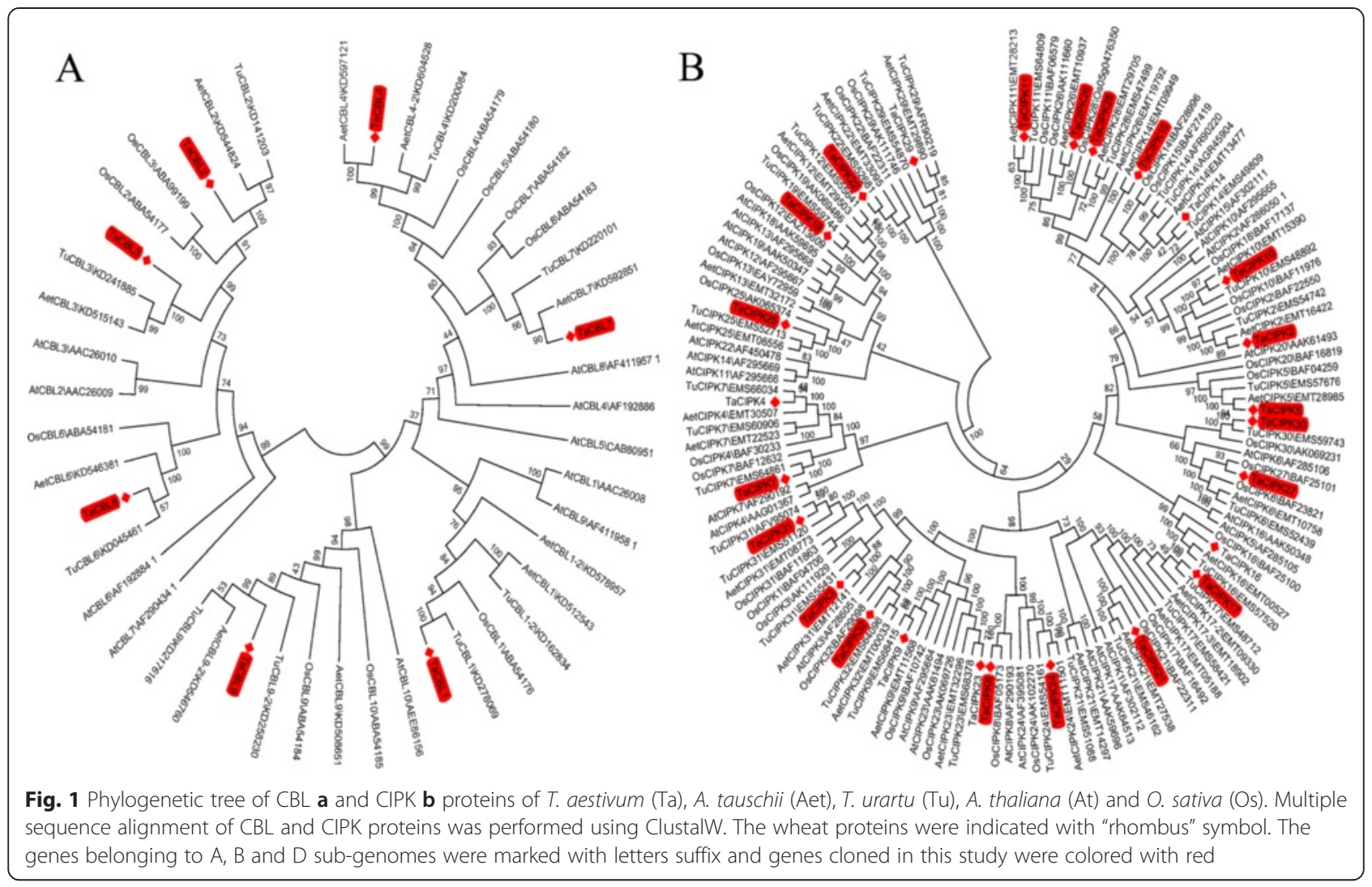

$T a C B L$ and $T a C I P K$ are provided in Additional file 3. To explore the evolutionary relationship of the CBL/CIPKs, two phylogenetic trees were generated based on all transcribed CBL/CIPKs from T. aestivum, T. urartu, $A$. tauschii, $A$. thaliana, and $O$. sativa. As expected, the genes from $T$. aestivum, $T$. urartu, and $A$. tauschii had the closest evolutionary relationships, and these genes had closer phylogenetic relationships with rice (a monocot) than with Arabidopsis (a dicot). Thus, the names assigned to the identified genes in wheat followed their homology to rice CBLs and CIPKs (Fig. 1). To analyze the structural characteristics of TaCBL and TaCIPK genes, the gene structures including exons and introns were mapped based on the wheat genome sequence (Additional file 4). Some genes' structures were partly characterized with exons/intros, because of lack adequate genome sequences. Only the structures covering the ORF were regarded as complete structures and used for further analysis. All the TaCBLs were intron-rich, with five to eight introns. Among the TaCIPKs, 26 TaCIPKs had no introns, and the other 15 TaCIPKs had 5-14 introns. For most genes, there were no obvious differences in gene structure among genomes A, B, and D (e.g., TaCIPK3, TaCIPK10 and TaCIPK23). Some genes (like TaCIPK31, TaCBL9) had a different intron length between the structures in different genome locations.
The CBL proteins containing EF-hand motifs are known as $\mathrm{Ca}^{2+}$-binding proteins, and show similarities to $\mathrm{CML}$ and $\mathrm{CaM}$ proteins. Each of the seven TaCBL proteins harbored four EF-hand motifs. Although these motifs in CBLs did not shared high similarity compared with CaM and CML proteins in other species (Arabidopsis, rice, C.elegans, yeast, zebrafish, mouse, and human), they were conserved among the CBLs in Arabidopsis, rice, and wheat (Additional file 5). As for the TaCIPK proteins, all 20 TaCIPKs (the genes were amplified by PCR) had domain structures similar to that of AtSOS2; that is, these $20 \mathrm{TaCIPKs}$ harbored a protein kinase catalytic domain (PKC) and the NAF/FISL motif. Considering that CIPK proteins belong to the SnRK superfamily, we selected some representative SnRK superfamily proteins for comparisons of their structural features. As shown in Additional file 6, the PKC domains in SnRK family proteins were conserved and had some highly consistent sites, especially in the activation loop, while the NAF/ FISL motif was unique to CIPK proteins.

Identification and validation of TaCBL-TaCIPK interactions We analyzed the physical interactions between TaCBL and TaCIPK proteins in wheat using the $\mathrm{Y} 2 \mathrm{H}$ method. As shown in Table 2 (Additional file 7), the interactions between TaCBLs and TaCIPKs showed different strengths 
Table 2 Interactions of TaCIPKs with TaCBLs in yeast two-hybrid assay

\begin{tabular}{|c|c|c|c|c|c|c|c|}
\hline & TaCBL1-BD & TaCBL2-BD & TaCBL3-BD & TaCBL4-BD & TaCBL6-BD & TaCBL7-BD & TaCBL9-BD \\
\hline TaCIPK3-AD & + & + & + & + & - & - & - \\
\hline TaCIPK5-AD & + & + & - & + & + & + & - \\
\hline TaCIPK7-AD & - & - & - & - & - & - & - \\
\hline TaCIPK8-AD & - & - & + & - & - & - & - \\
\hline TaCIPK10-AD & - & - & - & - & - & - & - \\
\hline TaCIPK11-AD & - & + & + & - & + & - & + \\
\hline TaCIPK14-AD & + & + & + & + & + & + & - \\
\hline TaCIPK15-AD & $+*$ & + & + & $+^{*}$ & $+^{*}$ & + & - \\
\hline TaCIPK17-AD & - & + & + & - & - & - & - \\
\hline TaCIPK19-AD & - & - & - & - & - & - & - \\
\hline TaCIPK21-AD & - & + & + & - & + & + & - \\
\hline TaCIPK22-AD & - & - & - & - & - & + & - \\
\hline TaCIPK24-AD & - & - & - & - & - & - & - \\
\hline TaCIPK25-AD & + & + & - & - & - & - & - \\
\hline TaCIPK26-AD & - & + & + & + & - & + & - \\
\hline TaCIPK27-AD & - & + & $+^{*}$ & - & + & $-*$ & - \\
\hline TaCIPK28-AD & - & - & - & - & - & - & - \\
\hline TaCIPK29-AD & - & - & - & - & - & - & - \\
\hline TaCIPK31-AD & - & $+^{*}$ & + & + & - & + & $+^{*}$ \\
\hline TaCIPK32-AD & - & + & + & - & - & - & - \\
\hline
\end{tabular}

The interaction analyses of wheat TaCBL and TaCIPK proteins were performed by $\mathrm{Y} 2 \mathrm{H}$ method. TaCIPKs and TaCBLs were respectively cloned to PGAD and PGBK Vector, and then co-transformed into Y187 strains. The transformants containing the target plasmid combinations were grown on selection medium (TDO: SD/-Trp/-Leu/-His/+10 mM $3 \mathrm{AT})$ and indicated as growth (+, interaction) and no growth (-, no interaction). " ${ }^{\prime * \prime}$ represents the interactions were verified by BiFC assays

and specificities. TaCBL1 strongly interacted with five CIPKs (TaCIPK3, 5, 14, 15, and 25). TaCBL2 strongly interacted with 11 CIPKs (TaCIPK3, 5, 11, 14, 15, 17, 21, $25,26,27$, and 32). TaCBL3 showed strong interactions with 10 TaCIPKs (TaCIPK3, 8, 11, 14, 15, 17, 21, 26, 27, and 31) out of 19 TaCIPKs. TaCBL4, the ortholog of AtSOS3 (CBL4), strongly interacted with six TaCIPKs (TaCIPK3, 5, 14, 15, 26, and 31). TaCBL6 strongly interacted with five TaCIPKs (TaCIPK5, 11, 15, 21, and 27), and TaCBL7 interacted with seven TaCIPKs (TaCIPK5, 14, $15,21,22,26$, and 31). TaCBL9 strongly interacted with only two TaCIPK proteins (TaCIPK11 and TaCIPK31). To view the interactions from a different perspective, we found that TaCIPK7, 10, 19, 22, 24, 28, and 29 did not interact with any of the seven TaCBL proteins assayed, implying that these CIPK proteins might perceive signals from other unidentified CBL proteins in wheat. We selected nine of the interactions (CBL1-CIPK15, CBL2-CIPK31, CBL3-CIPK15, CBL3-CIPK27, CBL4CIPK15, CBL4-CIPK31, CIPK6-CIPK15, CBL7-CIPK27, and CBL9-CIPK31) detected between CBL and CIPK proteins using the $\mathrm{Y} 2 \mathrm{H}$ assay for further analyses in planta using the BiFC method (Additional file 8). For all of the assayed TaCBL-TaCIPK interaction complexes, yellow fluorescence signals were observed when TaCBLs and TaCIPKs were co-expressed in epidermal cells of tobacco leaves. Notably, it seemed that the observed preferential interactions were inconsistent with the close phylogenetic relationships of the TaCIPKs, namely, the closely phylogenetic related CIPK pairs do not display same interaction profile. For example, TaCIPK14 and TaCIPK15, which had $81 \%$ similarity, showed similar interactions with five TaCBLs (TaCBL1, 2, 3, 4 and 7) but different interactions with TaCBL6 and 9. Compared with TaCIPK14/15, other TaCIPKs showed lower amino acid sequence similarity and more diverse interaction specificity. Thus, sequence similarity or phylogenetic relationships are not sufficient to predict the result of CBL-CIPK interactions in the $\mathrm{Y} 2 \mathrm{H}$ assay.

Previous studies have shown that the NAF/FISL motif located in the $C$-terminal regulatory domain and the kinase activity of the PKC domain in the $\mathrm{N}$-terminal region of CIPKs are necessary and sufficient for mediating interactions with CBLs $[5,6,33]$. The roles of the redundant parts (those other than the NAF/FISL motif) in the $C$-terminal region of CIPKs have not yet been fully studied. Here, the TaCIPK11 protein, which interacted with CBL2, 3, 6, and 9, contained a large $C$-terminal region (amino acids 311-507) that was not present in the other CIPKs. This led us to speculate about the role of this large fragment in regulating 
the specific interactions of this protein. Analyses of a series of $C$-terminal deletion mutants of TaCIPK11 (CIPK11-M1, M2, M3, and M4; M1-M3 containing the complete NAF/ FISL motif, and M4 with a truncated NAF/FISL motif) showed that there were diverse interaction patterns between TaCIPK11 mutants and TaCBLs (Fig. 2a). CBL3, 6, and 9 could not interact with any of the TaCIPK11 deletion mutants, while TaCBL1/TaCBL4 physically interacted with some or all of the mutants. TaCBL2 lost its specific interaction with the minimal mutant (CIPK11-M4). TaCBL7 did not interact with TaCIPK11 or any of its mutants. It seems that TaCBL3, 6 , and 9 require the last 86 amino acid

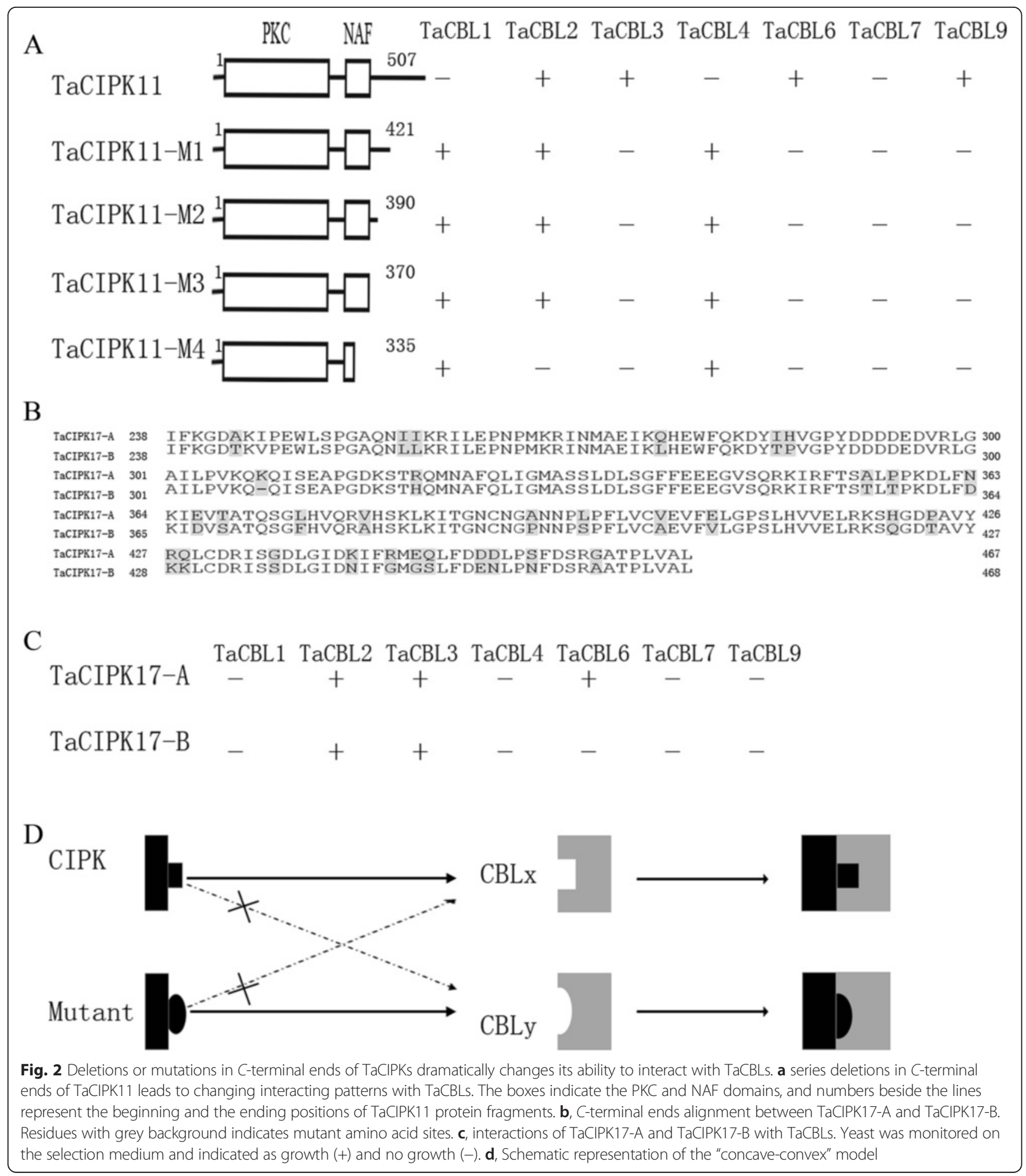


for interaction with TaCIPK11 and also inhibits the binding of TaCBL1 and TaCBL4. These changes in the patterns of interaction may result from changes in protein structure after the deletions. In terms of this deletion assay, we supposed that the interaction between CBL and CIPK followed a "concave-convex" model as shown in Fig. 2d, similar to the model for the structures of AtSOS3-AtSOS2 and AtCBL2-AtCIPK14 complexes proposed by Sánchez et al. [34]. In this model, the spatial structures of CBLs/CIPKs have an important role for their interactions, and a consummate physical interaction depends on whether the NAF/FISL motif properly reach the CBL cavity. It is believed that the diversified spatial structures resulted from divergent $C$-terminal region of CIPKs explain the molecular basis of the selectivity of certain CBLs towards particular CIPKs. This led to another intriguing question; that is, do the nonsynonymous sequence variations of alleles from different wheat sub-genomes result in aberrant spatial structures that affect the patterns of interaction? To answer this question, we amplified TaCIPK17- $A$, which encodes a protein with many amino acid variations in the $C$-terminal regulatory region compared with the homologous TaCIPK17-B protein (Fig. 2b). Interestingly, TaCIPK17-A not only interacted with TaCBL2 and TaCBL3, but also with TaCBL6 in Y2H experiments (Fig. 2c). Despite two mutant sites $\left(G^{23}\right.$ to $A^{23}, G^{48}$ to $\left.D^{48}\right)$ were observed at PKC domain, it is possible that several amino acid variations in the $C$-terminal regulatory region would confer different patterns of interaction. Such changes in interactions may render CIPKs capable of perceiving signals from other CBLs. These results indicate that the TaCBL-TaCIPK signaling pathway in wheat is very complex because of its allohexaploid genome.

\section{Expression patterns of $T a C B L$ and TaCIPK genes at different developmental stages in wheat}

To investigate the spatial expression patterns of TaCBLs and TaCIPKs in wheat, we detected their transcriptional levels using RT-PCR in 10 representative tissues, i.e., coleoptile, root (seedling and flowering stage), stem (seedling and flowering stage), leaf (seedling and flowering stage), flag leaf (flowering stage), pistil, and stamen (Fig. 3d). The results showed that the majority of TaCBLs and TaCIPKs were expressed at different levels in all tissues tested. Most $T a C B L$ genes were constitutively expressed in all organs and at all developmental stages, although the transcript levels of certain genes were very low in some tissues. Some CIPK genes were expressed abundantly in certain tissues. For example, TaCIPK 22 was specifically expressed in the root at the seedling stage, indicating that it may play an important role in this organ. Moreover, the gene transcript levels differed between vegetative (seedling) and flowering stages. There were higher transcript levels of TaCBL3 in seedlings than in plants at the flowering stage (roots, stems, and leaves). The flag leaf, as the last leaf of wheat, delivers the largest proportion of photosynthate to fill wheat grains [35]. Among the genes expressed in the flag leaf, TaCBL7 showed lower transcript levels in leaves of seedlings and plants at the flowering stage, and higher transcript levels in the flag leaf, implying that it might play roles in regulating photosynthesis and/or metabolism in the flag leaf. To study the transcription levels of TaCBLs and TaCIPKs during the whole life cycle of wheat, we analyzed microarray data that were obtained from tissues at various developmental stages; these data were obtained from publically accessible databases. The genes formed two groups according to their expression patterns (Fig. 3a). Group I consisted of the genes with high transcript abundance in nearly all tissues (TaCBL1, 2, and 6 and TaCIPK2, 8, 9, 15, 16, 23, 25, and 27). Group II genes consisted of 4 TaCBLs and 15 TaCIPKS that showed different expression patterns in different tissues and stages. Some of them showed low transcript levels in all tissues. For most of the TaCBLs/TaCIPKs, their expression profiles in the microarray data matched to the RT-PCR results, but a few genes showed inconsistencies. For example, high transcript levels of TaCBL4 were detected in the stamen in RT-PCR analyses, but low expression levels in anthers (before anthesis) were indicated in the microarray data. This inconsistency may have resulted from differences in the growth stages of plants between experiments, or from the different methods used.

To further clarify the detailed roles of TaCIPK and $T a C B L$ in seed germination, we analyzed public microarray data representing the transcription patterns during seed germination. As shown in Fig. 3b, TaCIPK6, 12, 14, $15,16,23,25$, and 27 , and $T a C B L 2$ were up-regulated $(\log >1, P<0.05)$ after $12 \mathrm{~h}$ of imbibition, and then down-regulated $(\log <1, P<0.05)$. TaCIPK12, TaCIPK31, $T a C I P K 32$, and $T a C B L 3$ were down-regulated during imbibition $(\log >1, P<0.05)$. Semi-quantitative RT-PCR analyses were conducted to confirm the $T a C B L$ and TaCIPK transcription patterns at five stages of germination $(0,12,24,36$, and $48 \mathrm{~h})$. All of the selected up-regulated genes were induced during seed germination (the EST of TaCIPK12 was unavailable, and the probe sequence of CIPK12 was not amplified), but the timing of induction differed (Fig. 3e). TaCIPK16, TaCIPK25, and TaCIPK27 showed peak transcript levels after $12 \mathrm{~h}$ of imbibition, while TaCIPK14 and TaCIPK23 showed peak transcript levels at $48 \mathrm{~h}$ and lower transcript levels at other time points. These transcriptional patterns indicated that the genes played roles at different stages of germination. High transcript levels of TaCIPK15 were detected only at $36 \mathrm{~h}$, when the radicle of seeds emerged and the coleoptile became visible. There were high transcript levels of TaCIPK16 at the initial stage of germination $(12 \mathrm{~h})$. For down-regulated genes (TaCBL3, TaCIPK31 and TaCIPK32) (Fig. 3b), our data 


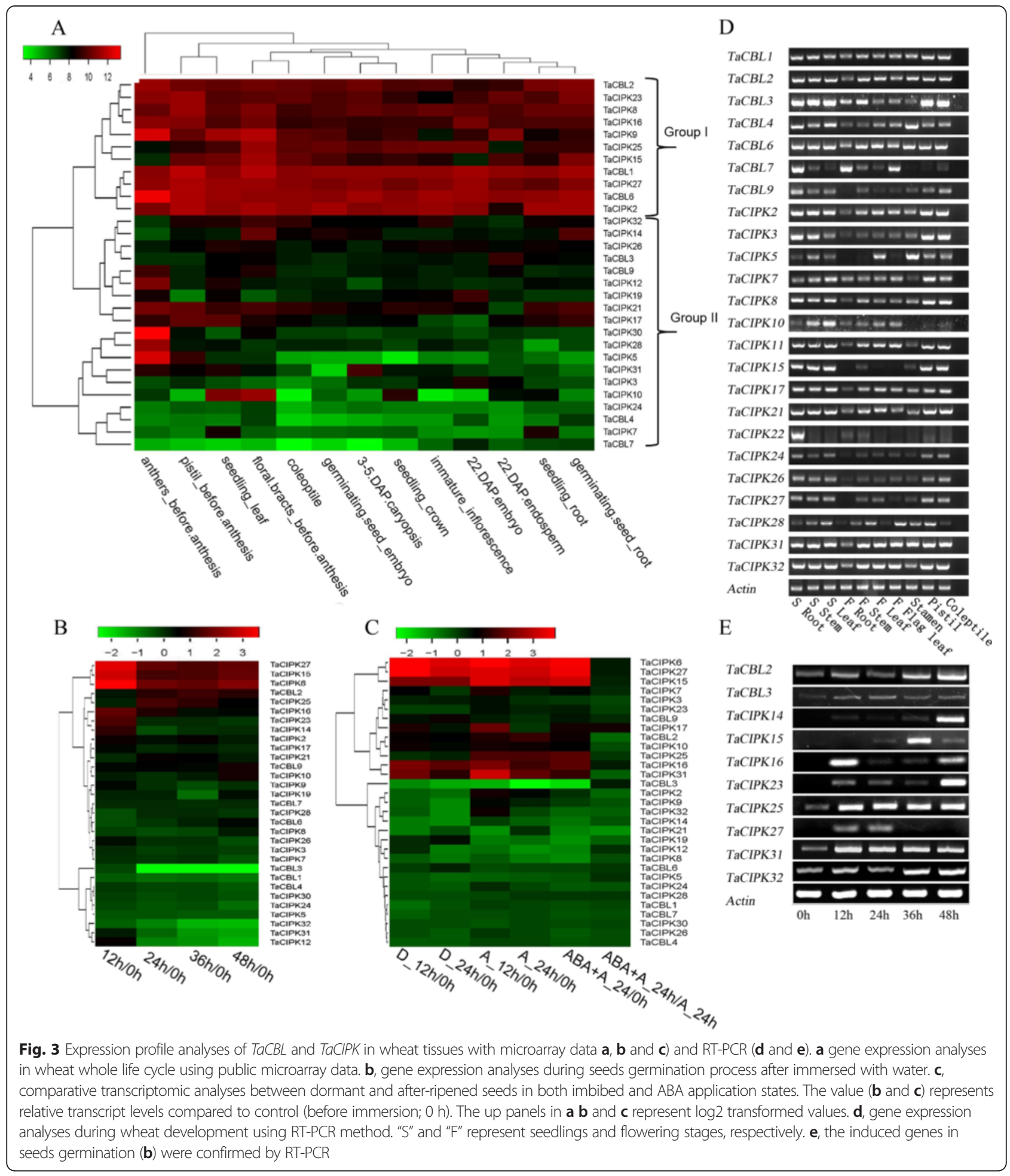

showed that these genes were actually up-regulated or showed no significantly changes in transcription. Moreover, despite some up-regulated genes were all validated in RTPCR and microarray data, they displayed peak transcript levels at different time point. For example, TaCIPK23 reached peak levels at $48 \mathrm{~h}$ in RT-PCR analysis, while it was up-regulated at $12 \mathrm{~h}$ followed with decreasing transcript levels in microarray analysis. The inconsistencies in the expression levels of genes may have been because the data were obtained using different methods, from different sub-species, or from seeds at slightly different stages of germination. We analyzed microarray data 
from another experiment, which focused on seed dormancy and after-ripening in bread wheat [36], and found that four genes (TaCIPK7, 10, 17, and 21) corresponded to germination and that TaCIPK31 was upregulated gene during germination (Fig. 3c). Interestingly, TaCIPK32 was down-regulated in dormant seeds, but upregulated in after-ripened seeds. These data provided the preliminary information about the roles of TaCBL-TaCIPK in seed germination.

\section{Expression profiles of $T a C B L$ and TaCIPK in response to abscisic acid and abiotic stresses}

There is considerable evidence that $C B L$ and $C I P K$ genes play important roles in the responses to phytohormones and abiotic stresses [10, 11, 37]. Among the stress hormones, ABA is a well-known signaling molecule in biotic or abiotic stress responses [38]. Cold, drought, and salinity are crucial factors affecting wheat growth and crop yields, and these stresses often cause oxidative stress.

To investigate the expression profiles of $T a C B L$ and $T a C I P K$ genes under stress conditions, we conducted realtime quantitative RT-PCR analyses. We analyzed the transcript levels of two TaCBLs and five TaCIPKs in wheat seedling roots and leaves under salt $(\mathrm{NaCl}), \mathrm{H}_{2} \mathrm{O}_{2}$, drought (PEG), and cold $\left(4^{\circ} \mathrm{C}\right)$ stress treatments, and in response to ABA application (Fig. 4). The transcript levels of all tested $T a C B L$ and TaCIPK genes changed in response to many of the treatments. The genes that did not show significant changes in transcript levels (significant criterion: $\log >2$, up-regulated; $\log <0.5$, down-regulated) were $T a C B L 9$ in the leaf (ABA/PEG treatments) and in the root (cold treatment), $\mathrm{TaCBL} 7$ in the root ( $\mathrm{NaCl}$ treatment), TaCIPK15 in the root $\left(\mathrm{H}_{2} \mathrm{O}_{2}\right.$ treatment), and TaCIPK31 in the leaf (ABA treatment). Among the analyzed genes, TaCIPK31 exhibited remarkable ABA-induced up-regulation in roots (350-fold increase compared with that in the control at $3 \mathrm{~h}$ ), but not in the leaves. Under salt stress, TaCIPK24 was up-regulated in roots and leaves. Therefore, it was selected for further functional characterization. The expression patterns of the analyzed TaCBLs and TaCIPKs were not completely consistent between seedling roots and leaves. For example, TaCBL4 was up-regulated in roots and down-regulated in leaves in response to ABA application. Several genes responded to the same stress; for example, TaCBL9, TCIPK7, 15, 24, and 32 were induced by $\mathrm{H}_{2} \mathrm{O}_{2}$. Among the five treatments, cold treatment resulted in the largest number of up-regulated genes, and none of the assayed genes were down-regulated by cold in roots or leaves. In brief, the expression patterns of the assayed genes differed among different treatments and tissues, indicating that various $\mathrm{TaCBLs}$ or $\mathrm{TaCIPKs}$ may participate in the signaling response to the same stress, and that a single $T a C B L$ or $\mathrm{TaCIPK}$ might function in multiple stress responses.
In previous studies, monocot and dicot species were shown to have divergent gene expression patterns in response to abiotic stresses or ABA application [11, 39]. Thus, we conducted a comparative expression analysis of $C B L s / C I P K s$ among wheat and two other important monocot crops (rice and maize). Microarray datasets of rice $C B L / C I P K$ expression patterns in response to abiotic stresses were collected by Zhang et al. [11], and a dataset of maize CIPK expression patterns was obtained by semiquantitative-PCR by Chen et al. [10]. The comparative analyses showed that several orthologous genes had similar transcription patterns. For example, the transcript level of CIPK32 increased in leaves and decreased in roots under salinity treatment in wheat and maize. However, some orthologous genes exhibited divergent expression patterns. For example, TaCBL4 showed increased transcript levels in roots and leaves under cold stress, while the transcript levels of OsCBL4 decreased under cold stress. Thus, there are both conserved and divergent expression models for orthologous genes among wheat, maize, and rice.

Next, we focused on stress-responsive cis-elements to decode the $C B L / C I P K$ expression models. These elements included the $\mathrm{ABA}$ responsive element (ABRE), the dehydration-responsive element (DRE)/C-repeat, and the low-temperature-responsive element (LTRE) [40-43]. Sequence analyses of 1-kbp sequences upstream from the start codon of the stress-inducible genes ( $T a C B L 4$, TaCBL9, TaCIPK7, TaCIPK15, and TaCIPK24) revealed that these genes contained putative ABRE, DRE, and LTRE elements (Additional file 9). These elements at least partly explained the stress-inducible expression patterns of these genes. However, even though TaCIPK31 and TaCIPK 32 were induced by ABA, PEG, and cold, they contained only the ABRE element in their promoter regions (Additional file 9). This result is not so surprising, since we only searched for three cis-elements in this study, and there may be other unidentified elements that are important for stress-responsive gene expression. Generally, the putative stress-responsive cis-elements in promoter regions are crucial for stress-responsive expression, and activation of the cis-elements depends on the tissue, the development stage, and/or the genetic background. Therefore, there are many explanations for the diversified expression patterns of orthologous genes among different species.

\section{Over-expression of TaCIPK24 improves salt tolerance in Arabidopsis}

Previous studies showed that AtCIPK24 (SOS2) and BnaCIPK24 (homolog of TaCIPK24) participated in the salt stress response in transgenic Arabidopsis [11, 44, 45]. In our expression analysis, TaCIPK 24 was induced in roots and leaves under salt stress (Fig. 4). Therefore, we generated transgenic Arabidopsis plants over-expressing TaCIPK24 


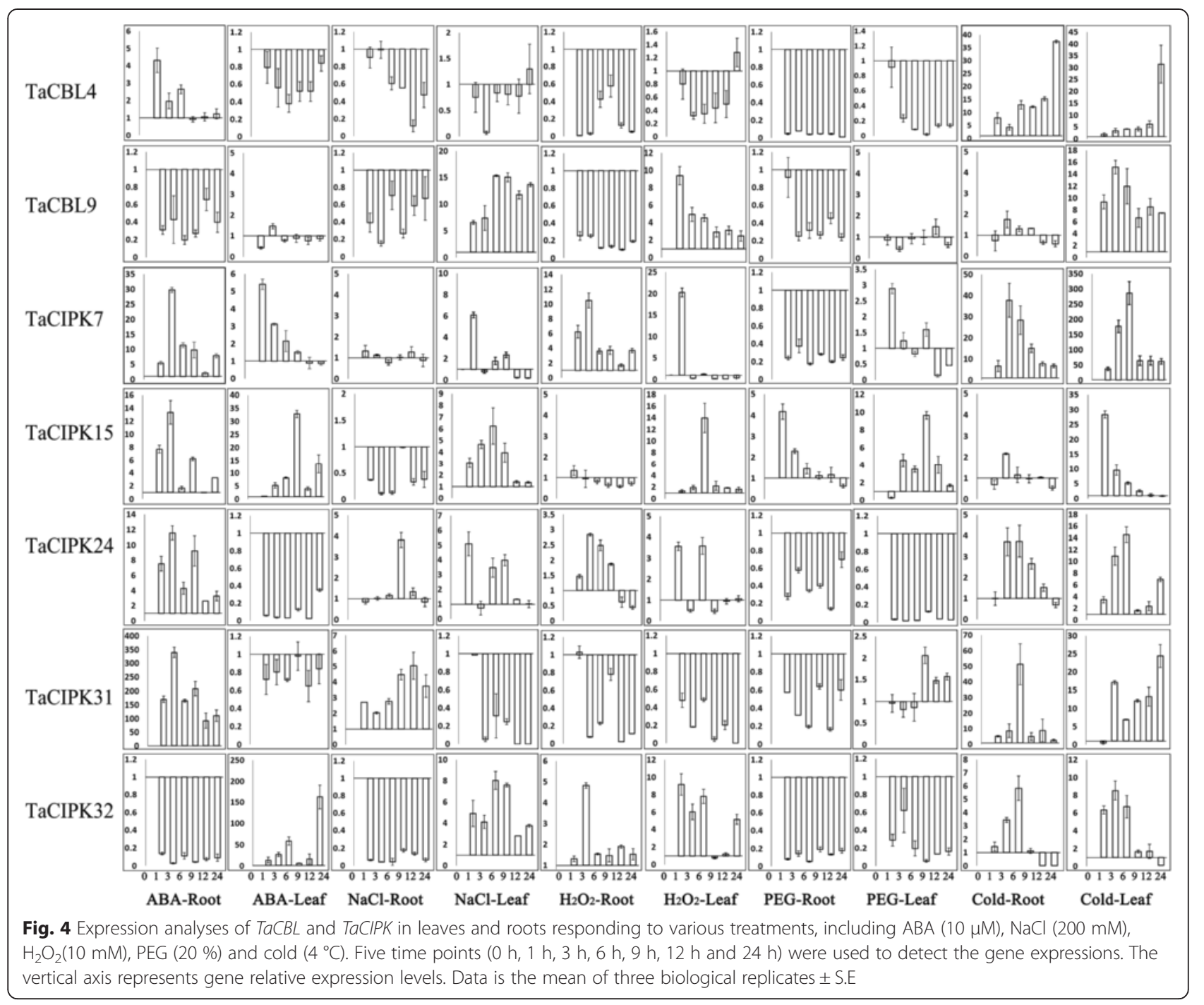

(or harboring pBI121 as the empty-vector control). In total, 28 transgenic lines were obtained. Three independent T3 homozygous lines (designated as OE-2, OE-7, and OE-9) were selected for further analyses. The transcript levels of TaCIPK24 were detected by RT-PCR (Fig. 5a). In plants grown on Murashige and Skoog (MS) medium, root growth did not differ significantly among the transformants, the empty-vector control, and wild type (Fig. $5 \mathrm{~b}$ and d). However, the 35S:TaCIPK24 transgenic plants showed greater root growth than those of controls after treatment with $150 \mathrm{mM} \mathrm{NaCl}$ (Fig. $5 \mathrm{c}$ and e; $p<0.05$ ). There were no significant differences in the phenotypes of aerial parts between transgenic lines and controls at the seedling stage under normal and salt-stress conditions. Therefore, our data indicated that TaCIPK24 promoted root elongation in transgenic plants under salt stress.

To further confirm the salt tolerance of TaCIPK24, seedlings of 10-day-old, soil-grown, wild-type and transgenic lines were irrigated with $200 \mathrm{mM} \mathrm{NaCl}$ for
25 d. As shown in Fig. 6a, the transgenic lines grew better than did the control plants. Furthermore, $\mathrm{Na}^{+}$ accumulated to lower levels in leaves of transgenic lines than in leaves of the empty-vector control and wild type. The $\mathrm{K}^{+}$contents were not significantly different between transgenic lines and the empty-vector control/ wild type (Fig. 6b), indicating that the improved salt tolerance may involve $\mathrm{Na}^{+}$efflux. In addition, excess $\mathrm{Na}$ ${ }^{+}$would generate reactive oxygen species (ROS), such as peroxide, superoxide, and hydrogen peroxide $\left(\mathrm{H}_{2} \mathrm{O}_{2}\right)$, causing oxidative stress in plants. The accumulation of $\mathrm{H}_{2} \mathrm{O}_{2}$ was reduced in transgenic lines compared with that in the control plants (Fig. 6c). This result indicated that decreased $\mathrm{Na}^{+}$contents led to lower ROS production (at least $\mathrm{H}_{2} \mathrm{O}_{2}$ ) and thus, the plants were able to grow well under salt stress conditions. Antioxidant enzymes are crucial for ROS scavenging and play roles in the salt stress response in plants. Therefore, we measured the activities of three important antioxidant enzymes (catalase, CAT; 


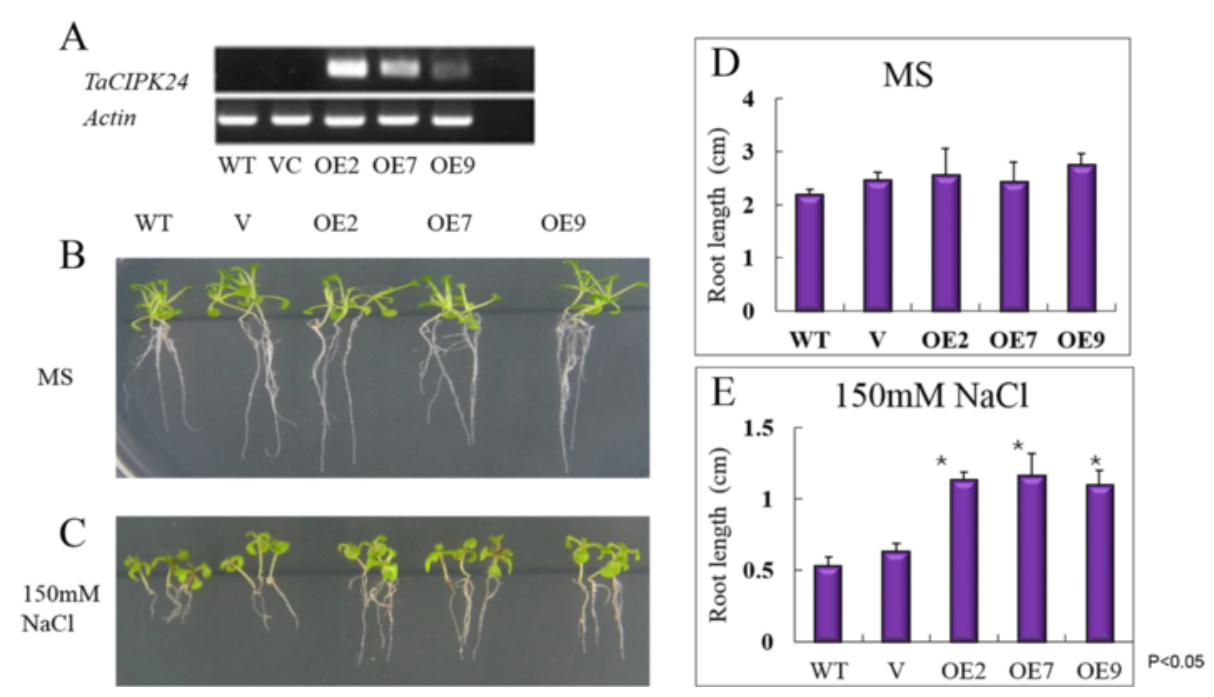

Fig. 5 Overexpression of TaCIPK24 in Arabidopsis. a TaCIPK24 expression levels in selected transgenic lines, vector control and wild type. b, c the plants grown on control (MS medium) and stress condition (MS + $150 \mathrm{mM} \mathrm{NaCl}$ ). d, e statistical analysis of the changing root length between MS control and salt stress condition $(p<0.05)$

peroxidase, POD; and superoxide dismutase, SOD) in transgenic plants and controls under salt stress and normal conditions. The activities of CAT, POD, and SOD were higher in transgenic lines than in wild-type and empty-vector controls (Fig. 6c). These results showed that the ectopic expression of TaCIPK24 enhanced salt tolerance through facilitating $\mathrm{Na}^{+}$efflux and ROS scavenging.

\section{Conclusions}

CBL and CIPK proteins play important roles in the $\mathrm{Ca}^{2+}$ signaling pathway and affect plant development, as well as participating in biotic and abiotic stress responses. $C B L$ s and CIPKs have been identified and functionally characterized in Arabidopsis, rice, maize, and canola [10-12, 46, 47], but less is known about the CBLs and $C I P K s$ in wheat. In this study, we identified seven $T a C B L$ and 29 TaCIPK genes from wheat, and cloned cDNAs of seven TaCBLs and 20 TaCIPKs. Analyses of TaCBLTaCIPK identified 24 TaCBL and 79 TaCIPK loci in the wheat genome, indicating that there were large numbers of these gene loci in the hexaploid wheat. Blast analyses suggested that four $T a C B L$ and $55 T a C I P K$ loci were derived from the T. urartu and A. tauschii genomes. We studied the preferential interactions between TaCBL and TaCIPK proteins and found 44 CBL-CIPK interaction complexes. The proteins TaCIPK7, 10, 19, 22, 24, 28, and 29 did not interact with any of the seven TaCBL proteins assayed in these experiments. Expression analyses revealed that the majority of TaCBLs and TaCIPKs were expressed at varying levels in all tested tissues, although the transcript levels of some of these genes were very low in some tissues. We conducted RT-PCR analyses to monitor the transcript levels of seven $T a C B \mathrm{~L}$ and $17 \mathrm{TaCIPK}$ genes under abiotic stress and hormone treatments. Most were distinctly regulated by at least one treatment. Among them, TaCIPK24, the ortholog of Arabidopsis SOS2, was up-regulated by salt stress in root and leaf tissues. We generated transgenic Arabidopsis lines over-expressing TaCIPK24, and all of them showed enhanced salt tolerance. Overall, this study lays a solid foundation for further exploration of the roles of TaCBL and TaCIPK proteins in abiotic stress responses, and enhances our understanding the functions of the wheat TaCBL and TaCIPK gene families. These results also provide information that will be useful for the genetic manipulation of wheat to improve stress tolerance.

\section{Methods}

Identification of TaCBL and TaCIPK genes

The reported CBL and CIPK protein sequences from rice and Arabidopsis were used to search the NCBI UniGene database. All the selected EST sequences were collected to form a local database and were assembled into longer cDNA sequences. To further validate the integrity of $T a C B L$ and $T a C I P K$ gene sequences, these sequences were used to search the $T$. aestivum $\mathrm{cv}$. Chinese Spring draft genome using the BLASTn method at Gramene (http://www.gramene.org/) and IWGSC (http://www.wheatgenome.org/). Rigorous criteria $\left(\mathrm{E}<10^{-5}\right.$, identity $>90 \%$ ) were used to ensure the reliability of the nucleotide sequences.

The gene-specific primers used for gene cloning were designed using Primer Premier 6 software (http:// www.premierbiosoft.com/). TaCBLs and TaCIPKs were amplified by RT-PCR from cDNA mixtures of prepared 


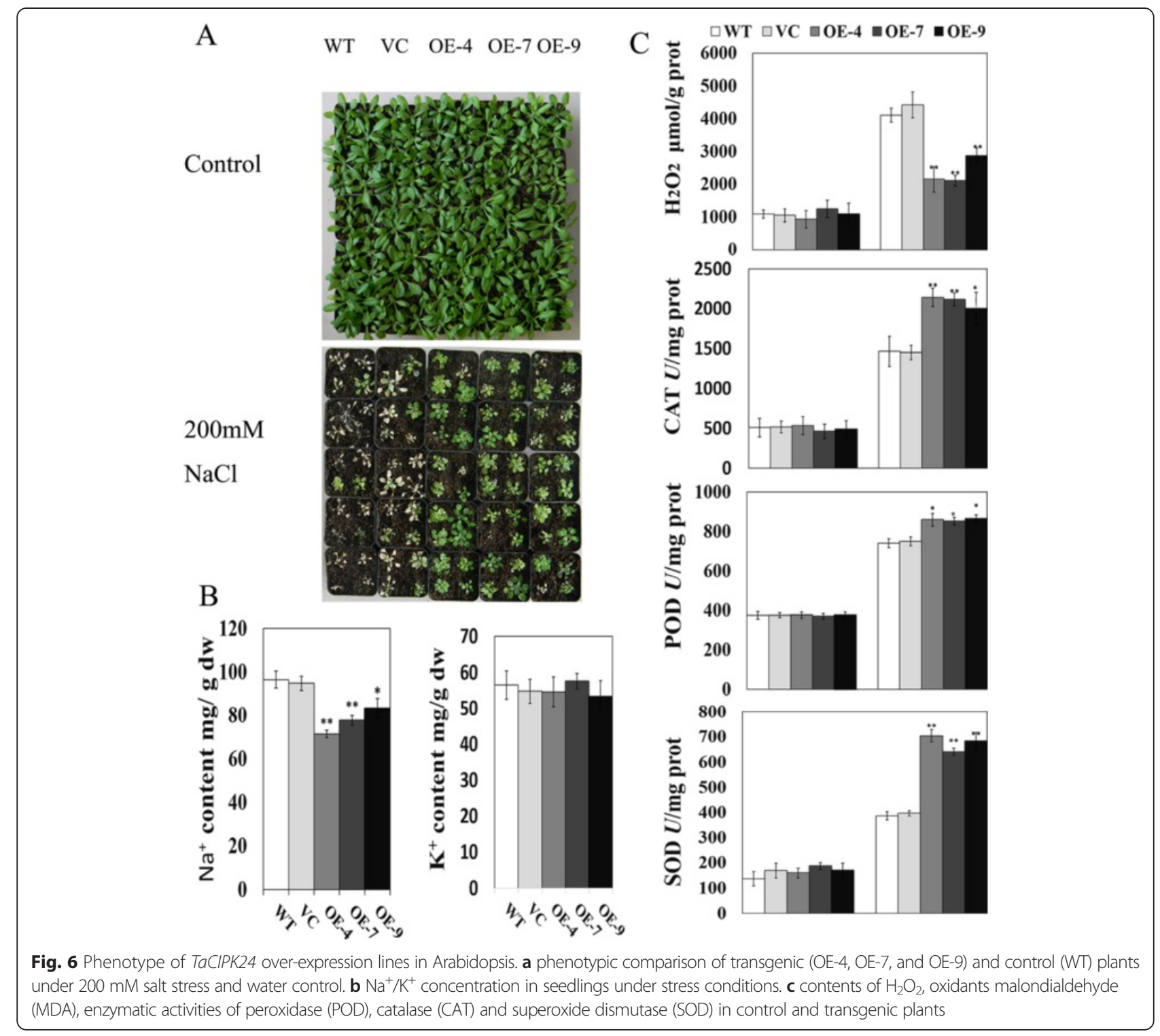

from wheat (cv. Chinese Spring) that included cDNAs from the coleoptile, root, stem, leaf, flag, leaf, pistil, anthers, and from seedlings treated with polyethylene glycol, $\mathrm{NaCl}$, cold, and ABA.

The structures of TaCBL and TaCIPK genes were illustrated using the Gene Structure Display Server (GSDS, http://gsds.cbi.pku.edu.cn/). Briefly, the cDNA sequences of T. aestivum, T. urartu, and A. tauschii were used to search the related genomic DNA sequences of $T$. aestivum following the instruction of GSDS. Multiple sequence alignments were executed using Clustal W (http://www.clustal.org) with default parameters. An unrooted neighbor-joining phylogenetic tree was constructed using MEGA 5.2.2 software (http://www.megasoftware.net). Genome-based sequences were used to confirm the affiliations of genes in all 21 chromosomes. Chromosomal locations were identified on genetic maps with Genome Zipper V5 at IWGSC and visualized by MapDraw. To analyze putative cis-elements in the promoters of $\mathrm{TaCBL} / \mathrm{TaCIPK}$ genes, $1-\mathrm{kbp}$ regions upstream of the CDS were extracted from wheat genomic sequences and subjected to a cis-element search using PLACE (http://www.dna.affrc.go.jp/PLACE/signalup.html).

\section{Gene expression analyses}

Wheat (T. aestivum L. cv. Chinese Spring) seeds were surface sterilized and then germinated in distilled water in a greenhouse at $25{ }^{\circ} \mathrm{C}$. The germinated seeds were collected at five different time points $(0 \mathrm{~h}, 12 \mathrm{~h}, 24 \mathrm{~h}, 36 \mathrm{~h}$, and $48 \mathrm{~h})$ to analyze gene expression during seed germination. After 2 weeks, young seedlings were subjected to $A B A(10 \mu \mathrm{M})$, $\mathrm{H}_{2} \mathrm{O}_{2}(10 \mathrm{mM})$, cold $\left(4{ }^{\circ} \mathrm{C}\right), \mathrm{NaCl}(200 \mathrm{mM})$, and PEG 
$(20 \% \mathrm{v} / \mathrm{v})$ treatments for $24 \mathrm{~h}$. Samples were collected at $0 \mathrm{~h}, 1 \mathrm{~h}, 3 \mathrm{~h}, 6 \mathrm{~h}, 9 \mathrm{~h}, 12 \mathrm{~h}$, and $24 \mathrm{~h}$ after the stress treatments for analyses. Leaf samples for analyses of CAT, POD, and SOD activities were collected at $25 \mathrm{~d}$ after the stress treatments. All the samples were harvested, frozen in liquid nitrogen, and stored at $-80{ }^{\circ} \mathrm{C}$ until use. Total RNA was isolated from frozen tissue using a Plant total RNA Extraction kit (ZOMANBIO, http://www.zomanbio.com). The reverse-transcription reactions were performed using a FastQuant RT kit (TIANGEN, http://www.tiangen.com/).

The primers used for expression analyses are listed in Additional file 2. The microarray datasets were processed using R 3.0.1 with the "Affy" package. Expression intensities were first normalized using the arithmetic RMA normalization method and then used for expression analyses. TaCBL and TaCIPK DNA sequences were used to probe the Affymetrix microarray datasets with blastn in PLEXdb (http://www.plexdb.org/index.php). The microarray expression data and gene probes used for these analyses are listed in Additional file 10.

\section{Yeast two-hybrid (Y2H) and bimolecular fluorescence complementation (BiFC) assays}

The $\mathrm{Y} 2 \mathrm{H}$ assays were conducted using the MatchMaker yeast two-hybrid system (www.clontech.com). The ORF regions of $T a C B L$ and $T a C I P K$ were respectively sub-cloned into pGBKT7 and pGADT7 vectors and co-transformed into the Y187 yeast strain. The transformants were grown on double-dropout medium (DDO: SD/-Trp/-Leu) and selected on triple-dropout medium (TDO: SD/-Trp/-Leu/-His) containing $10 \mathrm{mM}$ 3-amino-1, 2, 4-triazole (3-AT). For BiFC assays, the ORF regions of TaCBL and TaCIPK were sub-cloned into 35S-SPYCE and 35S-SPYNE vectors, respectively. After confirmation by sequencing, these constructs were separately transformed into Agrobacterium tumefaciens GV3101, then into tobacco leaves by Agrobacterium infiltration. Freshly transformed Agrobacterium cell cultures were re-suspended in suspension medium (10 mM MES- $\mathrm{KOH}$ (pH 5.6), $10 \mathrm{mM} \mathrm{MgCl}_{2}$, and $0.1 \mathrm{mM}$ acetosyringone), adjusted to an $\mathrm{OD}_{600}$ of $0.5-0.8$, and left at room temperature for $3 \mathrm{~h}$ before infiltration into tobacco leaves. Infiltrated leaf discs were collected 3-5 d later for observation under a confocal microscope. The TaCBLs and TaCIPKS primers used for vector construction are listed in Additional file 2: Table S1.

\section{Arabidopsis transformation and treatments}

The full-length coding sequence of TaCIPK24 was inserted into the pBI121 overexpression vector. Arabidopsis plants were transformed using the floral-dip method with $A$. tumefaciens strain EHA105 [48]. The assayed plants (transgenic Arabidopsis lines and wild type) were grown on MS medium until they reached the four-leaf stage, then transferred onto MS medium containing $\mathrm{NaCl}(150 \mathrm{mM})$ for salt stress analysis. The seedling root lengths were measured 7 days later. For salt tolerance analysis, 10-dayold soil-grown plants were irrigated with $200 \mathrm{mM} \mathrm{NaCl}$ at $5 \mathrm{~d}$ intervals for $25 \mathrm{~d}$.

Ion accumulation, $\mathrm{H}_{2} \mathrm{O}_{2}$ content, and enzyme activity measurements

To determine ion contents, samples were analyzed by atomic absorption spectrometry. Briefly, leaves were dried at $80{ }^{\circ} \mathrm{C}$ for $3 \mathrm{~d}$, mixed with $30 \%(\mathrm{v} / \mathrm{v}) \mathrm{H}_{2} \mathrm{O}_{2}$, heated for $15 \mathrm{~min}$ at $180{ }^{\circ} \mathrm{C}$, and then digested with concentrated $\mathrm{HNO}_{3}$ overnight. The samples were then analyzed using an atomic absorption spectrometer (AA-6300, Shimadzu Corporation). The $\mathrm{H}_{2} \mathrm{O}_{2}$ content and activities of CAT, POD, and SOD were measured by spectrophotometric methods using commercial detection kits (A064, A007, A084, and A001, Nanjing Jiancheng, China).

\section{Availability of supporting data}

All the supporting data are included as additional files.

\section{Additional files}

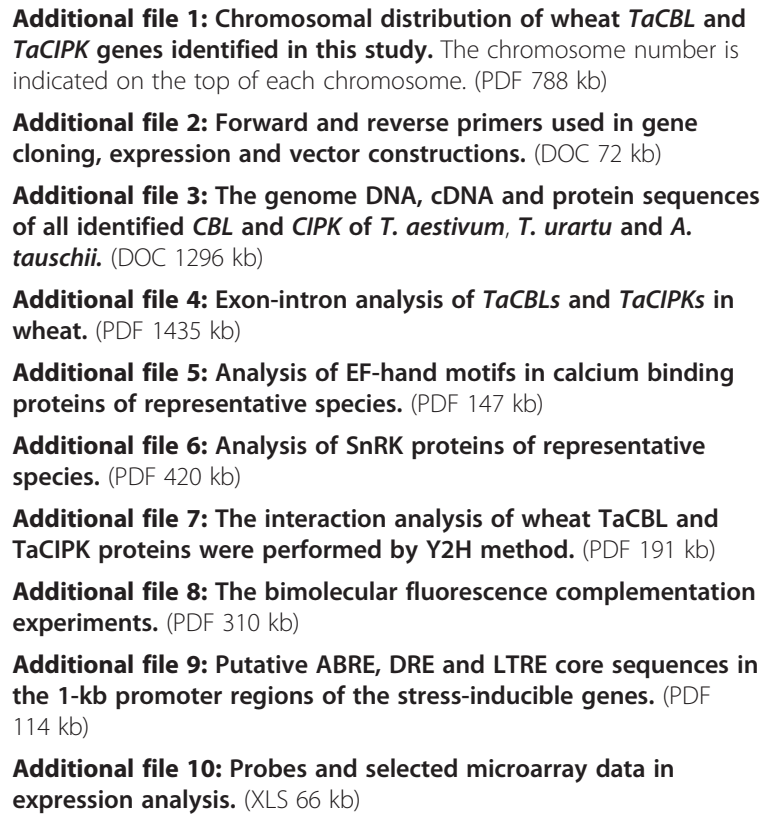

Additional file 10: Probes and selected microarray data in expression analysis. (XLS $66 \mathrm{~kb}$ )

\section{Abbreviations}

ABA: Abscisic acid; ABRE: ABA responsive element; BiFC: bimolecular fluorescence complementation; CBL: Calcineurin B-like protein; CDPK: calcium-dependent protein kinase; CIPK: CBL interacting protein kinase; CML: calmodulins, calmodulin-like protein; DDO: double-dropout medium; DRE: dehydration-responsive element; EST: expressed sequence tag; $\mathrm{H}_{2} \mathrm{O}_{2}$ : hydrogen peroxide; LTRE: low-temperature-responsive element; MS: Murashige and Skoog medium; POD: peroxidase; qRT-PCR: quantitative real-time polymerase chain reaction; ROS: reactive oxygen species; SOD: superoxide dismutase; SOS: salt overly sensitive; TDO: triple-dropout medium; VC: vector control; Y2H: yeast two-hybrid. 


\section{Competing interests}

The authors declare that they have no competing interests.

\section{Authors' contributions}

GYH, GXY and TS conceived and designed the experiments. TS and YW participated in all experiments, analyzed the data. MW, TTL, XTW, SYW and YZ performed the gene cloning, RT-PCR, Y2H analyses, genetic transformation and physiological assays. TS, GYH and GXY wrote the manuscript. All authors read and approved the manuscript.

\section{Acknowledgements}

This work was supported by International S \& T Cooperation Key Projects of MoST (grant No. 2009DFB30340); National Genetically Modified New Varieties of Major Projects of China (2015ZX08002-004, 2015ZX08010-004); Research Fund for the Doctoral Program of Higher Education of China (Grant no. 2012014211075). We thank Prof. Jörg Kudla (Universität Münster, Germany) for providing the BiFC vectors.

\section{Received: 22 September 2015 Accepted: 30 October 2015} Published online: 04 November 2015

\section{References}

1. Sheen J. $\mathrm{Ca}^{2+}$-dependent protein kinases and stress signal transduction in plants. SCIENCE. 1996;274(5294):1900-2.

2. Snedden WA, Fromm H. Calmodulin, calmodulin-related proteins and plant responses to the environment. Trends Plant Sci. 1998;3(8):299-304.

3. Albrecht V, Weinl S, Blazevic D, D'Angelo C, Batistic O, Kolukisaoglu U, et al. The calcium sensor CBL1 integrates plant responses to abiotic stresses. Plant J. 2003;36(4):457-70.

4. Shi J, Kim KN, Ritz O, Albrecht V, Gupta R, Harter K, et al. Novel protein kinases associated with calcineurin B-like calcium sensors in Arabidopsis. Plant Cell. 1999;11(12):2393-405.

5. Albrecht V, Ritz O, Linder S, Harter K, Kudla J. The NAF domain defines a novel protein-protein interaction module conserved in $\mathrm{Ca}^{2+}$-regulated kinases. EMBO J. 2001;20(5):1051-63.

6. Hashimoto K, Eckert C, Anschutz U, Scholz M, Held K, Waadt R, et al. Phosphorylation of calcineurin B-like (CBL) calcium sensor proteins by their CBL-interacting protein kinases (CIPKs) is required for full activity of CBLCIPK complexes toward their target proteins. J Biol Chem. 2012;287(11):7956-68.

7. Lin H, Yang Y, Quan R, Mendoza I, Wu Y, Du W, et al. Phosphorylation of SOS3-like calcium binding protein8 by SOS2 protein kinase stabilizes their protein complex and regulates salt tolerance in Arabidopsis. Plant Cell. 2009;21(5):1607-19.

8. Du W, Lin H, Chen S, Wu Y, Zhang J, Fuglsang AT, et al. Phosphorylation of SOS3-like calcium-binding proteins by their interacting SOS2-like protein kinases is a common regulatory mechanism in Arabidopsis. Plant Physiol. 2011;156(4):2235-43.

9. Kudla J, Xu Q, Harter K, Gruissem W, Luan S. Genes for calcineurin B-like proteins in Arabidopsis are differentially regulated by stress signals. P NATL ACAD SCI USA. 1999;96(8):4718-23.

10. Chen X, Gu Z, Xin D, Hao L, Liu C, Huang J, et al. Identification and characterization of putative CIPK genes in maize. J Genet Genomics. 2011;38(2):77-87.

11. Zhang $H$, Yang B, Liu WZ, Li H, Wang L, Wang B, et al. Identification and characterization of CBL and CIPK gene families in canola (Brassica napus L). BMC Plant Biol. 2014;14:8

12. Zhao J, Sun Z, Zheng J, Guo X, Dong Z, Huai J, et al. Cloning and characterization of a novel CBL-interacting protein kinase from maize. Plant Mol Biol. 2009;69(6):661-74.

13. Qiu QS, Guo Y, Dietrich MA, Schumaker KS, Zhu JK. Regulation of SOS1, a plasma membrane $\mathrm{Na}^{+} / \mathrm{H}^{+}$exchanger in Arabidopsis thaliana, by SOS2 and SOS3. Proc Natl Acad Sci U S A. 2002;99(12):8436-41.

14. Batelli G, Verslues PE, Agius F, Qiu Q, Fujii H, Pan S, et al. SOS2 promotes salt tolerance in part by interacting with the vacuolar $\mathrm{H}^{+}$-ATPase and upregulating its transport activity. Mol Cell Biol. 2007;27(22):7781-90.

15. Kim BG, Waadt R, Cheong YH, Pandey GK, Dominguez-Solis JR, Schultke S, et al. The calcium sensor CBL10 mediates salt tolerance by regulating ion homeostasis in Arabidopsis. Plant J. 2007;52(3):473-84.
16. Ren XL, Qi GN, Feng HQ, Zhao S, Zhao SS, Wang Y, et al. Calcineurin B-like protein CBL10 directly interacts with AKT1 and modulates $\mathrm{K}^{+}$homeostasis in Arabidopsis. Plant J. 2013;74(2):258-66.

17. Xu J, Li HD, Chen LQ, Wang Y, Liu LL, He L, et al. A protein kinase, interacting with two calcineurin B-like proteins, regulates $\mathrm{K}^{+}$transporter AKT1 in Arabidopsis. Cell. 2006;125(7):1347-60.

18. Li J, Long Y, Qi GN, Li J, Xu ZJ, Wu WH, et al. The Os-AKT1 channel is critical for $\mathrm{K}+$ uptake in rice roots and is modulated by the rice CBL1-CIPK23 complex. Plant Cell. 2014;26(8):3387-402.

19. Grefen C, Blatt MR. Do calcineurin B-like proteins interact independently of the serine threonine kinase CIPK23 with the $\mathrm{K}^{+}$channel AKT1? Lessons learned from a menage a trois. Plant Physiol. 2012;159(3):915-9.

20. Liu LL, Ren HM, Chen LQ, Wang Y, Wu WH. A protein kinase, calcineurin B-like protein-interacting protein Kinase 9 , interacts with calcium sensor calcineurin B-like Protein3 and regulates potassium homeostasis under low-potassium stress in Arabidopsis. Plant Physiol. 2013;161(1):266-77.

21. Tang RJ, Liu H, Yang Y, Yang L, Gao XS, Garcia VJ, et al. Tonoplast calcium sensors CBL2 and CBL3 control plant growth and ion homeostasis through regulating V-ATPase activity in Arabidopsis. Cell Res. 2012;22(12):1650-65.

22. Eckert C, Offenborn JN, Heinz T, Armarego-Marriott T, Schultke S, Zhang C, et al. The vacuolar calcium sensors CBL2 and CBL3 affect seed size and embryonic development in Arabidopsis thaliana. Plant J. 2014;78(1):146-56.

23. Kim KN, Cheong YH, Grant JJ, Pandey GK, Luan S. CIPK3, a calcium sensorassociated protein kinase that regulates abscisic acid and cold signal transduction in Arabidopsis. Plant Cell. 2003;15(2):411-23.

24. de la Torre F, Gutierrez-Beltran E, Pareja-Jaime Y, Chakravarthy S, Martin GB, Del PO. The tomato calcium sensor Cbl10 and its interacting protein kinase Cipk6 define a signaling pathway in plant immunity. Plant Cell. 2013;25(7):2748-64.

25. Piao HL, Xuan YH, Park SH, Je BI, Park SJ, Park SH, et al. OsCIPK31, a CBLinteracting protein kinase is involved in germination and seedling growth under abiotic stress conditions in rice plants. Mol Cells. 2010;30(1):19-27.

26. Ok SH, Cho JH, Oh SI, Choi MN, Ma JY, Shin JS, et al. Calcineurin B-like 3 calcium sensor associates with and inhibits 5'-methylthioadenosine nucleosidase 2 in Arabidopsis. Plant Sci. 2015;238:228-40.

27. Jia J, Zhao S, Kong X, Li Y, Zhao G, He W, et al. Aegilops tauschii draft genome sequence reveals a gene repertoire for wheat adaptation. Nature. 2013;496(7443):91-5.

28. Ling HQ, Zhao S, Liu D, Wang J, Sun H, Zhang C, et al. Draft genome of the wheat A-genome progenitor Triticum urartu. Nature. 2013;496(7443):87-90.

29. Brenchley R, Spannagl M, Pfeifer M, Barker GL, D'Amore R, Allen AM, et al. Analysis of the bread wheat genome using whole-genome shotgun sequencing. Nature. 2012:491(7426):705-10.

30. Deng X, Hu W, Wei S, Zhou S, Zhang F, Han J, et al. TaCIPK29, a CBLinteracting protein kinase gene from wheat, confers salt stress tolerance in transgenic tobacco. Plos One. 2013;8(7), e69881.

31. Deng X, Zhou S, Hu W, Feng J, Zhang F, Chen L, et al. Ectopic expression of wheat TaCIPK14, encoding a calcineurin B-like protein-interacting protein kinase, confers salinity and cold tolerance in tobacco. Physiol Plant. 2013;149(3):367-77.

32. Petersen G, Seberg O, Yde M, Berthelsen K. Phylogenetic relationships of Triticum and Aegilops and evidence for the origin of the A, B, and D genomes of common wheat (Triticum aestivum). Mol Phylogenet Evol. 2006;39(1):70-82.

33. Guo Y, Halfter U, Ishitani M, Zhu JK. Molecular characterization of functional domains in the protein kinase SOS2 tha is required for plant salt tolerance. Plant Cell. 2001;13(6):1383-400.

34. Sanchez-Barrena MJ, Martinez-Ripoll M, Albert A. Structural Biology of a Major Signaling Network that Regulates Plant Abiotic Stress: The CBL-CIPK Mediated Pathway. Int J Mol Sci. 2013;14(3):5734-49.

35. Borrill $P$, Fahy $B$, Smith AM, Uauy C. Wheat grain filling is limited by grain filling capacity rather than the duration of flag leaf photosynthesis: a case study using NAM RNAi plants. Plos One. 2015;10(8), e134947.

36. Liu A, Gao F, Kanno Y, Jordan MC, Kamiya Y, Seo M, et al. Regulation of wheat seed dormancy by after-ripening is mediated by specific transcriptional switches that induce changes in seed hormone metabolism and signaling. Plos One. 2013;8(2), e56570.

37. Yu Q, An L, Li W. The CBL-CIPK network mediates different signaling pathways in plants. Plant Cell Rep. 2014;33(2):203-14.

38. Xiong L, Schumaker KS, Zhu JK. Cell signaling during cold, drought, and salt stress. PLANT CELL. 2002;14(Suppl):S165-83. 
39. Liu T, Ohashi-Ito K, Bergmann DC. Orthologs of Arabidopsis thaliana stomatal $\mathrm{bHLH}$ genes and regulation of stomatal development in grasses. Development. 2009;136(13):2265-76.

40. Yamaguchi-Shinozaki K, Shinozaki K. Organization of cis-acting regulatory elements in osmotic- and cold-stress-responsive promoters. Trends Plant Sci. 2005;10(2):88-94.

41. Shinwari ZK, Nakashima K, Miura S, Kasuga M, Seki M, Yamaguchi-Shinozaki $K$, et al. An Arabidopsis gene family encoding DRE/CRT binding proteins involved in low-temperature-responsive gene expression. Biochem Bioph Res Co. 1998;250(1):161-70.

42. Simpson SD, Nakashima K, Narusaka Y, Seki M, Shinozaki K, YamaguchiShinozaki K. Two different novel cis-acting elements of erd1, a clpA homologous Arabidopsis gene function in induction by dehydration stress and dark-induced senescence. Plant J. 2003;33(2):259-70.

43. Xue GP. Characterisation of the DNA-binding profile of barley HvCBF1 using an enzymatic method for rapid, quantitative and high-throughput analysis of the DNA-binding activity. Nucleic Acids Res. 2002;30(15), e77.

44. Liu J, Ishitani M, Halfter U, Kim CS, Zhu JK. The Arabidopsis thaliana SOS2 gene encodes a protein kinase that is required for salt tolerance. Proc Natl Acad Sci U S A. 2000;97(7):3730-4.

45. Liu WZ, Deng M, Li L, Yang B, Li H, Deng H, et al. Rapeseed calcineurin Blike protein CBL4, interacting with CBL-interacting protein kinase CIPK24, modulates salt tolerance in plants. Biochem Biophys Res Commun. 2015.

46. Kolukisaoglu U, Weinl S, Blazevic D, Batistic O, Kudla J. Calcium sensors and their interacting protein kinases: genomics of the Arabidopsis and rice CBLCIPK signaling networks. Plant Physiol. 2004;134(1):43-58.

47. Xiang Y, Huang Y, Xiong L. Characterization of stress-responsive CIPK genes in rice for stress tolerance improvement. Plant Physiol. 2007;144(3):1416-28.

48. Clough SJ, Bent AF. Floral dip: a simplified method for Agrobacteriummediated transformation of Arabidopsis thaliana. Plant J. 1998;16(6):735-43.

\section{Submit your next manuscript to BioMed Central and take full advantage of:}

- Convenient online submission

- Thorough peer review

- No space constraints or color figure charges

- Immediate publication on acceptance

- Inclusion in PubMed, CAS, Scopus and Google Scholar

- Research which is freely available for redistribution 